\section{Kidney \\ Blood Pressure Research}

\title{
Long-Term Survival of Patients with IgA Nephropathy After Dialysis Therapy
}

\author{
Hiroyuki Komatsua Masao Kikuchia Hideto Nakagawa ${ }^{a}$ Akihiro Fukudaa

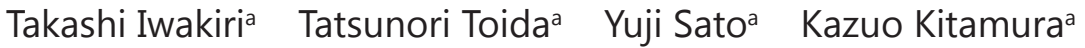 \\ Shouichi Fujimoto ${ }^{a, b}$
}

${ }^{a}$ First Department of Internal Medicine, University of Miyazaki Hospital; ${ }^{b}$ Department of Hemovascular Medicine and Artificial Organs, Faculty of Medicine, University of Miyazaki, Miyazaki, Japan

\section{Key Words}

IgA nephropathy $\cdot$ Cardio-cerebrovascular diseases $•$ End-stage kidney disease $•$ Dialysis

\begin{abstract}
Background/Aims: How dialysis affects the survival of patients with biopsy-proven IgA nephropathy (IgAN) is not fully understood. The present long-term cohort study quantifies the survival rates and incidence of cardio-cerebrovascular diseases (CCVDs) among such patients in Japan. Methods: Fifty-two of 433 patients with IgAN who had reached end-stage kidney disease underwent renal replacement therapy (RRT) between 1981 and 2010. The overall survival rate and incidence of CCVDs in these patients were evaluated during followup for $11.3 \pm 6.4$ years. Results: The mean age at starting RRT was $42.8 \pm 13.3$ years. Only seven patients died during follow-up (mortality rate, 1.2/100 person-years) and Kaplan-Meier analysis revealed favorable survival rates of $93.3 \%$ and $65.1 \%$ at 10 and 20 years, respectively, compared with that of patients with glomerulonephritis in the registry of the Japanese Society for Dialysis Therapy who required RRT. Malignancy and CCVDs were causes of death at $13.6 \pm$ 4.8 and $3.9 \pm 1.3$ years, respectively, after starting RRT. Fatal and non-fatal CCVDs developed in 15 (incidence, 2.7/100 person-years) patients and acute coronary syndrome and cerebral hemorrhage developed relatively soon after starting RRT. Cox proportional hazards models revealed that age at the time of starting RRT was a significant factor affecting the onset of CCVDs. Meanwhile, a history of having had corticosteroid as an initial treatment did not affect the onset of events. Conclusion: Although the survival of patients with IgAN is favorable after dialysis, the onset of CCVDs during the early phase of dialysis should be carefully monitored.
\end{abstract}




\section{Kidney \\ Blood Pressure Research}

\section{Introduction}

Immunoglobulin A nephropathy (IgAN) remains a leading cause of primary glomerulonephritis worldwide [1,2], accounting for $\sim 30 \%$ of the pathological diagnoses in the Japan Renal Biopsy Registry report for 2007 to 2008 [3]. Deterioration of renal function at biopsy, extensive histological damage, hypertension and proteinuria at biopsy and/or during the course are almost established prognostic factors, as $30 \%$ to $40 \%$ of all patients with IgAN reach end-stage kidney disease (ESKD) within 20 years [4]. These prognostic factors and outcomes from diagnosis to ESKD have been established based on the findings of many studies. However, little is known about prognosis after ESKD. Although the annual nationwide survey of the Japanese Society for Dialysis Therapy (JSDT) [5] describes survival rates for patients with primary diseases after dialysis, information about IgAN is not included.

Kidney disease has become recognized as a powerful risk factor for the progression of cardio-cerebrovascular diseases (CCVDs) and mortality, especially during the past decade [6]. Several reports indicate that the onset and continuance of IgAN increases the risk of CCVD events and mortality compared with the general population [7-9]. However, information about the incidence and risk factors for patients with IgAN undergoing renal replacement therapy (RRT) remains obscure.

Hence, this retrospective cohort study aimed to clarify the prognosis of patients who started RRT to treat IgAN diagnosed from renal biopsies and progressed to ESKD. We also investigated the incidence of CCVDs during RRT and risk factors affecting the onset of CCVDs during > 10 years of follow-up.

\section{Patients and Methods}

\section{Study design and patients}

Between 1981 and 2010, 433 patients at our institution were initially histologically diagnosed with IgAN based on light microscopic findings of proliferative changes in glomerular mesangial cells and immunofluorescence staining showing increased mesangial matrix associated with IgA and/or IgG and C3 deposition in renal biopsies. Patients with renal lesions caused by systemic diseases such as HenochSchönlein purpura nephritis, systemic lupus erythematosus, and liver cirrhosis were excluded. Among the 433 patients, 52 (12.0\%) had reached ESKD by February 2012 and 50 (96.2\%) of those were followed-up at 28 dialysis facilities or out-patient clinics in Miyazaki prefecture. Thus, sufficient medical information about the patients was available for retrospective investigation. Only two patients were lost to follow up after starting RRT.

\section{Outcomes and other evaluated factors}

The primary outcome of the study was the survival of the patients. Secondary outcomes comprised the onset of the following CCVDs: myocardial infarction (MI), unstable angina (UAP), aortic dissection, intracranial or subarachnoid hemorrhage $(\mathrm{CH} / \mathrm{SAH})$, cerebral infarction (CI) and peripheral artery disease (PAD). Hypertension was defined as systolic blood pressure (BP) > $140 \mathrm{mmHg}$ and/or diastolic BP > 90 $\mathrm{mmHg}$ or under treatment with antihypertensive drugs before diagnosis. A body mass index (BMI) $>25$ was defined as obese based on Japanese criteria [10]. RRT comprised hemodialysis (HD), peritoneal dialysis (PD) and renal transplant for patients with ESKD.

\section{Statistical analysis}

All continuous variables are presented as means \pm standard deviation (SD). Age at renal biopsy and starting RRT between CCVD and non-CCVD groups were compared using an unpaired t-test after the normal distribution was confirmed by Levene test. Differences in proportions were evaluated using the $\chi 2$ independent test or Fisher's exact test for $2 \times 2$ tables, depending on the number of categories. Survival rates of the two groups were analyzed using the Kaplan-Meier method, and differences were compared using the log-rank test. The impact of multiple covariates for the onset of CCVDs was assessed using Cox proportional hazards models. All independent variables included in multivariate analyses are expressed as categorical 


\section{Kidney \\ Blood Pressure Research}



Fig. 1. Survival rates of patients after dialysis therapy $(\mathrm{n}=50)$. Ten- and 20-year survival rates are $93.3 \%$ and $65.1 \%$, respectively.

\section{Kidney Blood Press Res 2013;37:649-656}

\begin{tabular}{l|l}
\hline DOI: $10.1159 / 000355745$ & (C) 2013 S. Karger AG, Basel
\end{tabular}

Published onlıne: December 14, 2013 www.karger.com/kbr

Table 1. Background of patients who reached end-stage kidney disease

\begin{tabular}{lc}
\hline Background & \\
\hline Total number of patients (n) & 52 \\
Sex (male / female) & $26 / 26$ \\
Age at renal biopsy (y) & $35.5 \pm 12.8$ \\
Systolic blood pressure at biopsy (mmHg) & $137.5 \pm 21.1$ \\
Diastolic blood pressure at biopsy (mmHg) & $84.9 \pm 13.9$ \\
Patients with hypertension at biopsy n(\%) & $25(48 \%)$ \\
Patients with BMI >25 at biopsy n(\%) & $9(17 \%)$ \\
Proteinuria at biopsy (g/day) & $2.67 \pm 1.71$ \\
Initial therapy at diagnosis & \\
$\quad$ RAS inhibitor n(\%) & $10(19 \%)$ \\
$\quad$ Steroid therapy n(\%) & $10(19 \%)$ \\
Other immunosuppressive therapy n(\%) & $0(0 \%)$ \\
Age starting RRT (y) & $42.8 \pm 13.3$ \\
Time from biopsy to RRT (y) & $7.2 \pm 5.3$ \\
Elapsed time from RRT to final observation (y) & $11.3 \pm 6.4$ \\
Fatalities n(\%) & $7(13 \%)$ \\
Onset of CVDDs n(\%) & $15(29 \%)$ \\
\hline CCVDs, cardio-cerebrovascular diseases; RRT: renal replacement \\
therapy. RAS: renin-angiotensin system; Data are shown as means \pm \\
SD or numbers and ratios of patients (n) (\%) \\
\hline
\end{tabular}

(coded as 0/1) or quantitative values. Sex, hypertension, BMI > 25, initial corticosteroid therapy, dialysis modality (HD vs. PD) and a history of renal transplantation were included as categorical variables. Age at starting RRT was included as a quantitative variable. The results of the multivariate analysis are expressed as hazards ratios with $95 \%$ confidence intervals (CI). A p value of $<0.05$ was considered significant for all data, which were statistically analyzed using SPSS for Windows, Advanced Statistical Release 15.0 (SPSS, Chicago, IL, USA).

\section{Results}

\section{Background factors and outcomes of patients}

Table 1 shows the background factors and outcomes of the patients who underwent dialysis. About half of all of the patients had hypertension at the time renal biopsies were obtained. Mean amounts of urinary protein of all patients was $2.67 \pm 1.71 \mathrm{~g} /$ day. Ten $(19.2 \%)$ of all patients were initially treated with renin-angiotensin system (RAS) inhibitors, and ten patients received steroid therapy. None of the patients were not administered immunosuppressive agents as an initial treatment. Age at starting RRT was $42.8 \pm 13.3$ years and the mean elapsed time from renal biopsy to RRT was 7.2 years. The initial modes of RRT among the 52 patients who progressed to ESKD were HD in $46(88.5 \%)$ and PD in $6(11.5 \%)$. Of the six patients who underwent renal transplantation during maintenance HD, four still had a renal graft at the final observation, whereas two had lost renal grafts and underwent further HD. Five patients eventually switched to HD therapy, and only one patient continued with PD therapy until the final observation among those who initially underwent PD. Seven $(13 \%)$ and $15(29 \%)$ patients respectively died and developed CCVDs during a mean followup period of $11.3 \pm 6.4$ years.

\section{Survival rates of patients with IgA nephropathy after starting RRT}

Figure 1 shows the survival rates of the 50 patients (excluding the two lost to followup). Kaplan-Meier curves revealed favorable 10- and 20-year survival rates of $93.3 \%$ and $65.1 \%$, respectively. Although none of the patients who started with PD died during the observation period, the survival rates did not significantly differ between those who started with PD or HD ( $\mathrm{p}=0.482$; log-rank test, data not shown). Table 2 compares prognoses between the JSDT registry and the present study. Chronic glomerulonephritis (CGN) in the JSDT registry includes primary glomerular diseases such as membranous nephropathy, 


\section{Kidney Blood Pressure Research}

Kidney Blood Press Res 2013;37:649-656

DOI: $10.1159 / 000355745$

Published onlıne: December 14, 2013

(C) 2013 S. Karger AG, Basel

Komatsu et al.: Survival of Patients with IgAN After Dialysis

Table 2. Comparison of prognosis between Japan dialysis registry data and present findings

\begin{tabular}{|c|c|c|c|c|}
\hline \multirow{4}{*}{$\begin{array}{l}\text { Primary disease of } \\
\text { dialysis patients } \\
\text { Observation periods (year) } \\
\text { Age starting RRT (year) }\end{array}$} & \multirow{2}{*}{$\begin{array}{l}\text { Present study } \\
\begin{array}{c}\text { IgAN } \\
(n=50)\end{array}\end{array}$} & \multicolumn{3}{|c|}{ Japan dialysis registry data* } \\
\hline & & \multirow{2}{*}{\multicolumn{2}{|c|}{$\begin{array}{l}\text { Chronic glomerulonephritis } \\
\text { (n = 205,111)** } \\
1983-2007\end{array}$}} & $\begin{array}{l}\text { All primary disease } \\
(\mathrm{n}=547,727)^{* *}\end{array}$ \\
\hline & $1986-2010$ & & & $1983-2007$ \\
\hline & 42.8 (mean) & $30-44$ (range) & 45 - 59 (range) & 51.9 - 66.8 (mean) \\
\hline 10 -year survival rate $(\%)$ & 93.3 & 83.8 & 63.7 & 36.3 \\
\hline 20-year survival rate $(\%)$ & 65.1 & 65.9 & 31.7 & 17.4 \\
\hline
\end{tabular}

membranoproliferative glomerulonephritis and focal segmental glomerulosclerosis as well as IgAN. The 10 and 20-year survival rates of the patients with IgAN (mean age, $42.8 \mathrm{y}$ ) were similar to those of younger (age, ranged from 30 to $44 \mathrm{y}$ ) patients with CGN, and was rather more favorable than that of older (age, ranged from 45 to $59 \mathrm{y}$ ) patients with CGN.

\section{Relationship between causes of death and period from start of RRT to death}

Seven (female $n=5$; male, $n=2$ ) patients died during a mean follow-up of $11.3 \pm 6.4$ years, and the mortality rate was 1.2/100 person-years. Three patients died of CCVDs (SAH, cardiac failure, cerebral hemorrhage), and three others died of malignant diseases (adult $\mathrm{T}$-cell leukemia, gastric cancer, renal cancer). The patient died from gastric cancer received long-term immunosuppressive therapy after the renal transplantation. The remaining patient died one month after surgery to replace a cardiac valve. The mean elapsed time from starting RRT was shorter for the three patients who died of CCVDs than for those who died of malignant diseases $(3.9 \pm 1.3$ vs. $13.6 \pm 4.8$ years, $p<0.05)$.

Onset of cardio-cerebrovascular diseases after patients with IgA nephropathy started RRT

Figure 2 shows age at diagnosis, at the start of RRT, and at the onset of CCVDs in six female and nine male patients. Over 10 years elapsed from diagnosis to the onset of CCVDs in three patients who were diagnosed as teenagers. Figure 3 shows when CCVDs developed after starting RRT in the fifteen patients. Seventeen CCVDs in 15 patients developed at an incidence of 2.7/100 person-years during the follow-up period. Acute coronary syndromes such as MI and UAP, and hemorrhagic strokes ( $\mathrm{CH} / \mathrm{SAH})$ tended to develop within five years of starting RRT, whereas CI tended to arise later. Univariate analyses showed that background factors at renal biopsy, a history of transplantation, and mode of RRT did not statistically differ between patients with $(n=15)$ and without $(n=35)$ CCVDs (Table 3). However, multivariate analysis using Cox proportional hazards models revealed that age at the time of starting RRT was a significant factor affecting the onset of CCVDs (HR, 1.06; 95\% C.I., 1.01-1.12; $\mathrm{p}=0.025)$. A history of initial corticosteroid therapy did not affect the onset of CCVDs (Table 4).

\section{Discussion}

The present findings revealed the following controversial issues: good survival rates after starting dialysis, relatively earlier onset of CCVDs than malignancies, more advanced age as a risk factor for CCVDs, and the absence of an influence of steroid therapy on CCVD onset in patients with IgAN after starting dialysis therapy.

The 10- and 20-year survival rates of the patients with IgAN after starting dialysis were $93.3 \%$ and $65.1 \%$, respectively, in this study, and these were similar to recent findings in Korea (88.6\% and 66.3\%) [9]. Several factors are thought to be involved in the favorable prognosis of patients with IgAN after starting dialysis. Firstly, such patients usually start dialysis at an 


\section{Kidney Blood Pressure Research}

Fig. 2. Types of cardio-cerebrovascular diseases (CCVDs) and elapsed time from diagnosis to CCVD onset. CH, cerebral hemorrhage; CI, cerebral infarction; MI, myocardial infarction; PAD, peripheral artery disease; RRT, renal replacement therapy; SAH, s ubara chnoid hemorrhage; UAP, unstable angina pectoris.

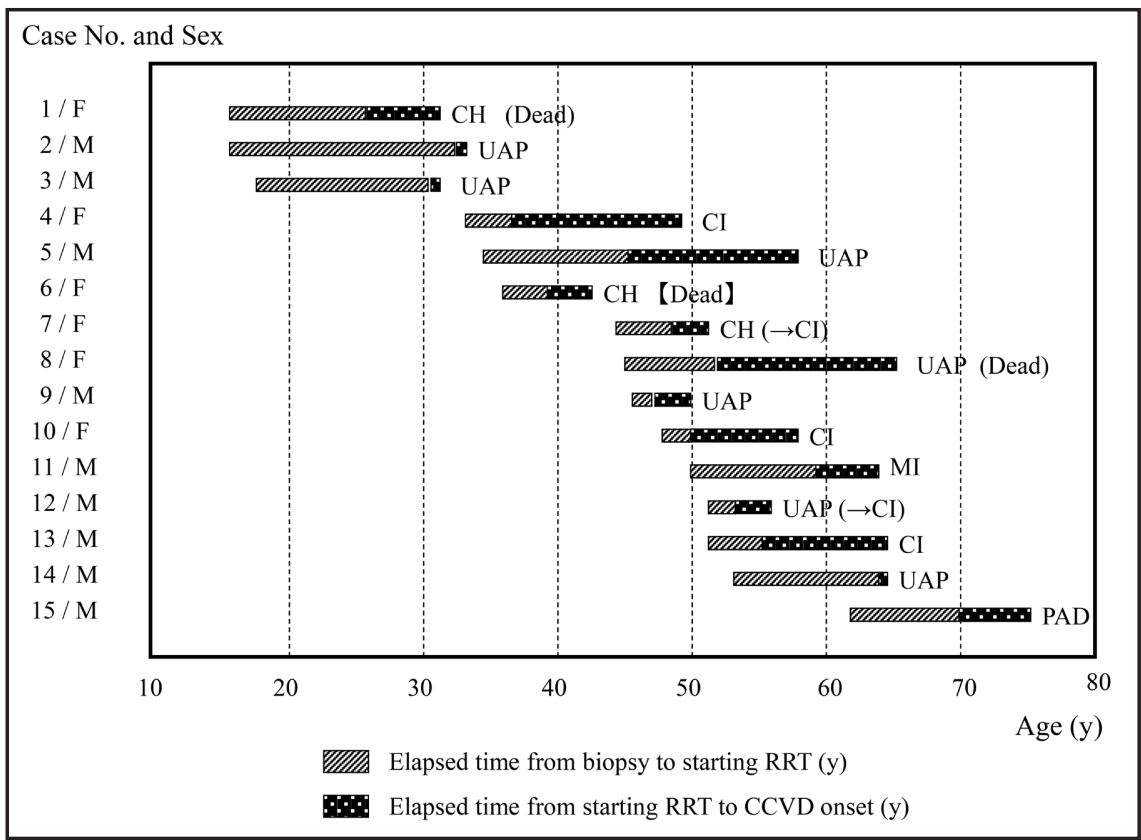

Fig. 3. Onset of cardio-cerebrovascular diseases (CCVDs) after starting dialysis therapy (17 events in 15 patients). Circles indicate onset of CCVDs. CH, cerebral hemorrhage; MI, myocardial infarction; PAD, peripheral artery disease; RRT, renal replacement therapy; SAH, subarachnoid hemorrhage; UAP, unstable angina pectoris.

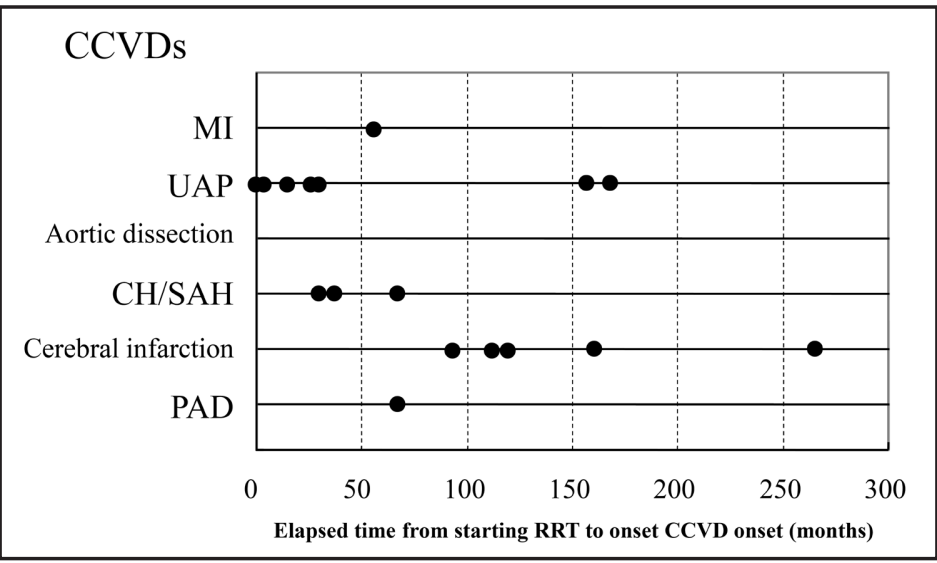

Table 3. Comparison of background factors between patients with and without cardio-cerebrovascular diseases on RRT

\begin{tabular}{lccc}
\hline Total patients $(\mathrm{n}=50)$ & CCVD $(-)$ & CCVD $(+)$ & $\mathrm{P}^{*}$ \\
\hline Sex (male / female) & $15 / 20$ & $9 / 6$ & 0.358 \\
Age at renal biopsy (y) & $34.3 \pm 11.8$ & $40.2 \pm 14.3$ & 0.132 \\
Patients with hypertension at biopsy $\mathrm{n}(\%)$ & $20(57 \%)$ & $5(33 \%)$ & 0.217 \\
Patients with BMI >25 at biopsy n(\%) & $4(11 \%)$ & $5(33 \%)$ & 0.106 \\
Age starting RRT (y) & $41.9 \pm 13.2$ & $47.1 \pm 12.6$ & 0.194 \\
History of steroid therapy & $9(26 \%)$ & $1(7 \%)$ & 0.246 \\
History of transplantation & $5(14 \%)$ & $1(7 \%)$ & 0.654 \\
RRT modality (HD/PD) & $30 / 5$ & $14 / 1$ & 0.654 \\
\hline *Unpaired test and Fisher's exact test; RRT, renal replacement therapy \\
\hline
\end{tabular}

earlier age than those who need dialysis for other reasons. Some observational studies have pointed out that starting dialysis younger is an important factor in surviving for up to 30 years $[11,12]$. In fact, the annual report of the JSDT registry (2008) states that the mean age of the entire dialysis patient population at that time was 65.3 years [5], whereas the mean age of the patients with IgAN in this study who started dialysis was 42.8 years. Secondly, IgAN might have a better prognosis than other types of primary glomerulonephritis. Chou et al. [13] reported that mortality rates were significantly higher for membranous nephropathy and focal segmental glomerulosclerosis than for IgAN (17.2\% and $14.4 \%$ vs. $4.6 \%)$ in an Asian cohort $(n=987)$ during a median follow-up period of 5.9 years. Lee et al. [14] also 


\section{Kidney Blood Pressure Research}

Kidney Blood Press Res 2013;37:649-656

DOI: 10.1159/000355745
Published onlıne: December 14, 2013

(C) 2013 S. Karger AG, Basel

www.karger.com/kbr

Table 4. Risk factors affecting onset of cardio-cerebrovascular diseases $(n=50)$

\begin{tabular}{llccc}
\hline Risk factors & & $\begin{array}{c}\text { Hazards } \\
\text { ratio }\end{array}$ & 95\% CI & P \\
\hline Age starting RRT & (per year) & 1.06 & $(1.01-1.12)$ & $0.025^{*}$ \\
Male & (vs. female) & 1.45 & $(0.41-5.10)$ & 0.561 \\
Hypertension at biopsy & (vs. normal BP) & 0.38 & $(0.11-1.29)$ & 0.121 \\
BMI $>25$ at biopsy & (vs. BMI < 25) & 1.58 & $(0.44-5.71)$ & 0.484 \\
History of steroid therapy & (vs. no therapy) & 0.88 & $(0.10-7.50)$ & 0.910 \\
History of transplantation & (vs. no transplantation) & 1.29 & $(0.12-13.7)$ & 0.834 \\
RRT by PD & (vs. HD) & 0.67 & $(0.08-5.37)$ & 0.705 \\
\hline
\end{tabular}

*Statistically significant

found that survival in a large cohort $(n=1,943)$ was better among patients with IgAN and than for those with focal segmental glomerulosclerosis. Our findings of a higher survival rate for IgAN than for other CGN (Table 2) are compatible with these data, even when the difference in age between the two groups is considered. On the other hand, the incidences of CCVDs and mortality are higher in patients with IgAN than in the general population. One study from Finland [7] found that $25 \%$ of patients with IgAN aged $>30$ years had some CCVDs at the end of follow-up, compared with only $9 \%$ of the general population. The Korean study [9] also showed that overall mortality including CCVDs was elevated by $43 \%$ in an age/sexmatched general population. Although IgAN is probably associated with a higher incidence of CCVDs and mortality than the general population, the risk seems to be lower than that of other types of CGN. This might be attributable to the unique features of IgAN, which is relatively limited to the kidneys and is associated with few other general complications.

Malignancy and CCVDs are the main causes of death among patients on dialysis. According to the JSDT registry in 2008 [5], cardiac failure (23.7\%) was the leading cause of death, and CCVDs (cerebrovascular disease and cardiac infarction, 12.7\%) and malignant tumors (9.2\%) were the third and fourth most common causes of death. The onset of fatal and non-fatal acute coronary syndromes (MI and UAP) and cerebral hemorrhage (CH/SAH) in the present study tended to occur relatively early (within 5 years), whereas that of death due to malignancy and non-fatal CI tended to occur over 10 years later. The CHOICE study [15], which surveyed the incidence and outcomes of cerebrovascular diseases in patients starting dialysis, found that the overall incidence of cerebrovascular events was 4.9/100 person-years, with ischemic stroke being the most common. That study also indicated that the rate of cerebrovascular events was the greatest during the first two years of dialysis therapy, and ischemic stroke accounted for the majority of events especially after this time frame. The results of the GRACE registry [16] indicated that patients with acute coronary syndrome who are on dialysis have a greater burden of established cardiac risk factors during the initial phase of dialysis, and obviously greater six-month mortality rates than patients who are not on dialysis. Although the incidence and mortality rates due to CCVDs in the present study were relatively low compared with previous reports $[5,15,16]$, careful monitoring of the onset of CCVDs is needed, especially during the early phase of dialysis. We believe that the main reason for the late onset of malignancy was that none of the patients had malignant disease at the start of dialysis in this study. Only one of three patients who died of malignancy underwent renal transplantation, which was required to continue immunosuppressive therapy. A larger study that excludes renal transplantation is needed to clarify the effect of dialysis on the onset of new malignancy.

The present study found that age at the time of starting RRT was a significant factor affecting the onset of CCVDs, whereas hypertension at diagnosis and a history of corticosteroid therapy was not. We confirmed that advanced age is an established risk factor for mortality and the onset of CCVDs $[17,18]$. The percentage of the hypertension at diagnosis was higher in non-CCVD patients than in CCVD patients ( $57 \%$ vs 33\%). Iseki et al. examined the relationship between BP and anti-hypertensive drugs therapy in HD patients, and found that one-year mortality rate was significantly lower in patients prescribed anti-hypertensive drugs than 


\section{Kidney \\ Blood Pressure Research}

in those non-prescribed. They refer to effect of the prescription of anti-hypertensive drugs on good prognosis of HD patients [19]. In fact, the percentage of the patients prescribed anti-hypertensive drugs was higher in non-CCVD patients than in CCVD patients in this study (66\% vs. 33\%). The existence of hypertension and use of anti-hypertensive drugs might counteract the each effect. Corticosteroid is considered as a possible risk factor for cardiovascular disease in patients with systemic lupus erythematosus [20]. Korkmaz et al. also implied that the prevalence of steroid therapy is higher among patients with systemic lupus erythematosus accompanied with, than without coronary atherosclerosis [21]. One reason for the discrepancy between IgAN and systemic lupus erythematosus might be the duration of steroid therapy. Long-term corticosteroid is generally needed as maintenance therapy for patients with systemic lupus erythematosus whereas that for IgAN is relatively short-term. In fact, the maximal duration of steroid therapy for our patients was three years.

This study has several limitations that require consideration. Firstly, this retrospective study at a single center comprised a small cohort, although the mean observation period was over 10 years. A multi-center study of a large cohort is needed to obtain more precise information about the incidence of CCVDs and mortality after dialysis in patients with IgAN. Secondly, information about each dialysis condition and medications such as antihypertensive drugs and erythropoietin stimulating agents, as well as the management of mineral-bone disorders during maintenance dialysis was unavailable. Although we conducted multivariate analyses, factors during maintenance dialysis were not included as independent factors. Finally, some race-related factors might be associated with the long survival and low incidence of CCVDs because all of our study patients were Japanese. In fact, rates of mortality and comorbid diseases differ among countries [19, 22].

\section{Conclusion}

The present findings suggested that Japanese patients with IgAN have a good prognosis, with low mortality rates and CCVD incidence after starting dialysis. Nevertheless, careful monitoring of CCVD onset is needed, especially during the early phase of dialysis. Further studies of a large cohort and an international comparison of mortality rates and CCVD incidence in patients with IgAN after dialysis therapy are required.

\section{Acknowledgements}

This study was presented in part at Kidney Week 2012 for the annual meeting of the American Society of Nephrology, 30.10.-04.11.2012, in San Diego, California, USA.

\section{Conflict of interest}

The authors have no conflicts of interest to disclose.

\section{References}

\footnotetext{
1 D’Amico G: The commonest glomerulonephritis in the world: IgA nephropathy. Q J Med 1987;64:709-727.

2 Julian BA, Waldo FB, Rifai A, Mestecky J: IgA nephropathy, the most common glomerulonephritis worldwide: A neglected disease in the United States? Am J Med 1988;84:129-132.

- Sugiyama H, Yokoyama H, Sato H, Saito T, Kohda Y, Nishi S, Tsuruya K, Kiyomoto H, Iida H, Sasaki T, Higuchi M, Hattori M, Oka K, Kagami S, Nagata M, Kawamura T, Honda M, Fukasawa Y, Fukatsu A, Morozumi K, Yoshikawa N, Yuzawa Y, Matsuo S, Kiyohara Y, Joh K, Taguchi T, Makino H: Committee for Standardization of Renal Pathological Diagnosis and Working Group for Renal Biopsy Database, Japanese Society of
} 


\section{Kidney \\ Blood Pressure Research}

Kidney Blood Press Res 2013;37:649-656

\begin{tabular}{l|l}
\hline DOI: $10.1159 / 000355745$ & (c) 2013 S. Karger AG, Basel \\
\hline
\end{tabular}

Publisnea onine: December 14, 2013

www.karger.com/kbr

Nephrology, Tokyo, Japan. Japan Renal Biopsy Registry: the first nationwide, web-based, and prospective registry system of renal biopsies in Japan. Clin Exp Nephrol 2011;15:493-503.

4 D'Amico G: Natural history of idiopathic IgA nephropathy and factors predictive of disease outcome. Semin Nephrol 2004;24:179-196.

-5 Nakai S, Suzuki K, Masakane I, Wada A, Itami N, Ogata S, Kimata N, Shigematsu T, Shinoda T, Syouji T, Taniguchi M, Tsuchida K, Nakamoto H, Nishi S, Nishi H, Hashimoto S, Hasegawa T, Hanafusa N, Hamano T, Fujii N, Marubayashi S, Morita O, Yamagata K, Wakai K, Watanabe Y, Iseki K, Tsubakihara Y: Overview of regular dialysis treatment in Japan (as of 31 December 2008). Ther Apher Dial 2010;14:505-540.

-6 Sarnak MJ, Levey AS, Schoolwerth AC, Coresh J, Culleton B, Hamm LL, McCullough PA, Kasiske BL, Kelepouris E, Klag MJ, Parfrey P, Pfeffer M, Raij L, Spinosa DJ, Wilson PW: American Heart Association Councils on Kidney in Cardiovascular Disease, High Blood Pressure Research, Clinical Cardiology, and Epidemiology and Prevention. Kidney disease as a risk factor for development of cardiovascular disease: a statement from the American Heart Association Councils on Kidney in Cardiovascular Disease, High Blood Pressure Research, Clinical Cardiology, and Epidemiology and Prevention. Circulation 2003;108:21542169.

-7 Myllymäki J, Syrjänen J, Helin H, Pasternack A, Kattainen A, Mustonen J: Vascular diseases and their risk factors in IgA nephropathy. Nephrol Dial Transplant 2006;21:1876-1882.

8 Berthoux F, Mohey H, Laurent B, Mariat C, Afiani A, Thibaudin L: Predicting the risk for dialysis or death in IgA nephropathy. J Am Soc Nephrol 2011;22:7527-61.

-9 Lee H, Kim DK, Oh KH, Joo KW, Kim YS, Chae DW, Kim S, Chin HJ: Mortality of IgA nephropathy patients: a single center experience over 30 years. PLoS One 2012;7:e51225.

10 Takahashi H, Mori M: Characteristics and significance of criteria for obesity disease in Japan 2011. Nihon Rinsho 2013;71:257-261 (in Japanese).

11 Otsubo S, Otsubo K, Sugimoto H, Ueda S, Otsubo Y, Otsubo O, Yajima A, Yagi S, Kataoka H, Iwasaki T, Iwasa Y, Takahashi M, Aoki A, Ino J, Uchida K, Yumura W, Akiba T, Nitta K: Characteristics of patients on hemodialysis therapy for more than 30 years. Ther Apher Dial 2007;11:274-279.

-12 Morimoto S, Nishioka H, Morita T, Jo F, Someya K, Nakahigashi M, Kusabe M, Ueda H, Takahashi N, Iwasaka T, Maki K: Characteristics of 20-year survivors undergoing maintenance hemodialysis. Ther Apher Dial 2010;14:547-551.

13 Chou YH, Lien YC, Hu FC, Lin WC, Kao CC, Lai CF, Chiang WC, Lin SL, Tsai TJ, Wu KD, Chen YM: Clinical outcomes and predictors for ESRD and mortality in primary GN. Clin J Am Soc Nephrol 2012;7:1401-1408.

14 Lee H, Kim DK, Oh KH, Joo KW, Kim YS, Chae DW, Kim S, Chin HJ: Mortality and renal outcome of primary glomerulonephritis in Korea: observation in 1,943 biopsied cases. Am J Nephrol 2013;37:74-83.

15 Sozio SM, Armstrong PA, Coresh J, Jaar BG, Fink NE, Plantinga LC, Powe NR, Parekh RS: Cerebrovascular disease incidence, characteristics, and outcomes in patients initiating dialysis: the choices for healthy outcomes in caring for ESRD (CHOICE) study. Am J Kidney Dis 2009;54:468-477.

-16 Gurm HS, Gore JM, Anderson FA Jr, Wyman A, Fox KA, Steg PG, Eagle KA: Global Registry of Acute Coronary Events (GRACE) Investigators: Comparison of acute coronary syndrome in patients receiving versus not receiving chronic dialysis (from the Global Registry of Acute Coronary Events [GRACE] Registry). Am J Cardiol 2012;109:19-25.

17 Iseki K, Shinzato T, Nagura Y, Akiba T: Factors influencing long-term survival in patients on chronic dialysis. Clin Exp Nephrol 2004;8:89-97.

- 18 Goodkin DA, Bragg-Gresham JL, Koenig KG, Wolfe RA, Akiba T, Andreucci VE, Saito A, Rayner HC, Kurokawa K, Port FK, Held PJ, Young EW: Association of comorbid conditions and mortality in hemodialysis patients in Europe, Japan, and the United States: the Dialysis Outcomes and Practice Patterns Study (DOPPS). J Am Soc Nephrol 2003;14:3270-3277.

19 Iseki K, Shoji T, Nakai S, Watanabe Y, Akiba T, Tsubakihara Y: Committee of Renal Data Registry of the Japanese Society for Dialysis Therapy. Higher survival rates of chronic hemodialysis patients on antihypertensive drugs. Nephron Clin Pract 2009;113:c183-190.

20 Sinicato NA, da Silva Cardoso PA, Appenzeller S: Risk factors in cardiovascular disease in systemic lupus erythematosus. Curr Cardiol Rev 2013 1;9:15-19.

21 Korkmaz C, Cansu DU, Kaşifoğlu T: Myocardial infarction in young patients ( $<$ or =35 years of age) with systemic lupus erythematosus: a case report and clinical analysis of the literature. Lupus 2007;16:289-297.

22 Kuriyama S: Characteristics of the clinical practice patterns of hemodialysis in Japan in consideration of DOPPS and the NKF/DOQI guidelines. Clin Exp Nephrol 2008;12:165-170. 